\title{
On Pillai's problem with the Fibonacci and Pell sequences
}

\author{
Santos Hernández Hernández · Florian Luca · \\ Luis Manuel Rivera
}

Received: date / Accepted: date

Abstract Let $\left(F_{n}\right)_{n \geq 0}$ and $\left(P_{n}\right)_{n \geq 0}$ be the Fibonacci and Pell sequences given by the inicial conditions $F_{0}=0, F_{1}=1, P_{0}=0, P_{1}=1$ and the recurrences formulas $F_{n+2}=F_{n+1}+F_{n}, P_{n+2}=2 P_{n+1}+P_{n}$ for all $n \geq 0$, respectively. In this note we shall study the Pillai's type problem

$$
F_{n}-P_{m}=F_{n_{1}}-P_{m_{1}}
$$

in non-negative integer pairs $(n, m) \neq\left(n_{1}, m_{1}\right)$. We completely solve this equation.

Keywords Fibonacci, Pell sequences · Pillai's type problem · Linear form in logarithms

Mathematics Subject Classification (2000) 11B39, 11D45 · 11D61 · 11J86

\section{Introduction}

Let $\mathbf{U}:=\left(U_{n}\right)_{n \geq 0}$ and $\mathbf{V}:=\left(V_{n}\right)_{n \geq 0}$ be two linearly recurrent sequences of integers. Recently, the following variation of a problem of Pillai has been studied. Find all non-negative integer solutions $\left(n, m, n_{1}, m_{1}\right)$ of the equation

$$
U_{n}-V_{m}=U_{n_{1}}-V_{m_{1}}, \quad(n, m) \neq\left(n_{1}, m_{1}\right) .
$$

S. Hernández Hernández · L. M. Rivera

Unidad Académica de Matemáticas, Universidad Autónoma de Zacatecas, Campus II, Calzada Solidaridad entronque Paseo a la Bufa, C.P. 98000, Zacatecas, Zac. MEXICO

Tel.: +52-492-9229975

E-mail: shh@uaz.edu.mx, luismanuel.rivera@gmail.com

F. Luca

School of Mathematics, University of the Witwatersrand, Private Bag X3, WITS 2050, Johannesburg, SOUTH AFRICA; Max Planck Institute for Mathematics, Vivatsgasse 7, 53111 Bonn, GERMANY; Department of Mathematics, Faculty of Sciences, University of Ostrava, 30 Dubna 22, 70103 Ostrava 1, CZECH REPUBLIC E-mail: Florian.Luca@wits.ac.za 
In particular, find also all integers $c$ which can be written as the difference between an element of $\mathbf{U}$ and an element of $\mathbf{V}$ in at least two different ways. Pillai [12], studied this problem when $\mathbf{U}$ and $\mathbf{V}$ are the sequences of powers of $a$, and powers of $b$, respectively, where $a, b$ are two given coprime integers different than $0, \pm 1$. It has been shown in [6] that, under some technical but natural conditions, equation (1) has only finitely many non-negative integer solutions and all of them are effectively computable. This version of Pillai's problem was initiated in [8] by Ddamulira, Luca and Rakotomalala who studied equation (1) when $\mathbf{U}$ and $\mathbf{V}$ are the sequences of Fibonacci numbers and powers of 2, respectively. Many other particular cases have been studied. See, for example [5], [3], [7]. We recall that the Fibonacci sequence $\left(F_{n}\right)_{n \geq 0}$ is given by $F_{0}=0, F_{1}=1$ and the recurrence formula

$$
F_{n+2}=F_{n+1}+F_{n} \quad \text { for all } \quad n \geq 0 .
$$

Let $\left(P_{n}\right)_{n \geq 0}$ be the Pell sequence given by $P_{0}=0, P_{1}=1$, and the recurrence formula

$$
P_{n+2}=2 P_{n+1}+P_{n} \quad \text { for all } \quad n \geq 0 .
$$

Their first terms are,

$0,1,1,2,3,5,8,13,21,34,55,89,144,233,377,610,987,1597, \ldots$

and

$$
0,1,2,5,12,29,70,169,408,985,2378,5741,13860,33461, \ldots,
$$

respectively. In this note, we study another particular case of this problem, namely equation (1) with Fibonacci and Pell numbers. More precisely, we look at the equation

$$
F_{n}-P_{m}=F_{n_{1}}-P_{m_{1}}
$$

in integer pairs $(n, m) \neq\left(n_{1}, m_{1}\right)$. Since $F_{1}=F_{2}=1$, we assume that $n \neq 1, n_{1} \neq 1$. That is, whenever we think of 1 as a member of the Fibonacci sequence, we think of it as being $F_{2}$. Our result is then the following

Theorem 1 All non-negative integer solutions $\left(n, m, n_{1}, m_{1}\right)$ of $(2)$ with $n \neq 1, n_{1} \neq 1$ belong to the set

$$
\left\{\begin{array}{llll}
(2,1,0,0), & (2,2,0,1), & (3,1,2,0), & (3,2,0,0), \\
(3,2,2,1), & (4,1,3,0), & (4,2,2,0), & (4,2,3,1), \\
(4,3,0,2), & (5,2,4,0), & (5,3,0,0), & (5,3,2,1), \\
(5,3,3,2), & (6,3,4,0), & (6,3,5,2), & (6,4,2,3), \\
(7,3,6,0), & (7,4,2,0), & (7,4,3,1), & (7,4,4,2), \\
(9,5,5,0), & (11,6,8,2), & (16,9,3,0), & (16,9,4,1)
\end{array}\right\} .
$$

The set of integers $c$ admitting two representations as a difference between a Fibonacci and a Pell number in at least two different ways is

$$
\{-4,-2,-1,0,1,2,3,5,8,19\} \text {. }
$$


The representations of the above $c$ are

$$
\begin{aligned}
-4 & =F_{6}-P_{4}=F_{2}-P_{3} ; \\
-2 & =F_{4}-P_{3}=F_{0}-P_{2} ; \\
-1 & =F_{2}-P_{2}=F_{0}-P_{1} ; \\
0 & =F_{5}-P_{3}=F_{3}-P_{2}=F_{2}-P_{1}=F_{0}-P_{0} ; \\
1 & =F_{7}-P_{4}=F_{4}-P_{2}=F_{3}-P_{1}=F_{2}-P_{0} ; \\
2 & =F_{16}-P_{9}=F_{4}-P_{1}=F_{3}-P_{0} ; \\
3 & =F_{6}-P_{3}=F_{5}-P_{2}=F_{4}-P_{0} ; \\
5 & =F_{9}-P_{5}=F_{5}-P_{0} ; \\
8 & =F_{7}-P_{3}=F_{6}-P_{0} ; \\
19 & =F_{11}-P_{6}=F_{8}-P_{2} .
\end{aligned}
$$

\section{Tools}

The first one is a lower bound for a linear form in logarithms due to Matveev [11]. Let $\alpha$ be an algebraic number of degree $d$. Let $a$ be the leading coefficient of its minimal polynomial over $\mathbf{Z}$ and let $\alpha_{1}=\alpha, \ldots, \alpha_{d}$ denote the conjugates of $\alpha$. The Weil height of $\alpha$ is defined as

$$
h(\alpha)=\frac{1}{d}\left(\log a+\sum_{i=1}^{d} \log \max \left\{\left|\alpha_{i}\right|, 1\right\}\right) .
$$

The height has the following basic properties. For $\alpha, \beta$ algebraic numbers and $m \in \mathbf{Z}$, we have:

- $h(\alpha+\beta) \leq h(\alpha)+h(\beta)+\log 2$.

- $h(\alpha \beta) \leq h(\alpha)+h(\beta)$.

- $h\left(\alpha^{m}\right)=|m| h(\alpha)$.

Now let $\mathbf{L}$ be a real number field of degree $d_{\mathbf{L}}, \alpha_{1}, \ldots, \alpha_{\ell} \in \mathbf{L}$ and $b_{1}, \ldots, b_{\ell} \in \mathbf{Z} \backslash\{0\}$. Let $B \geq \max \left\{\left|b_{1}\right|, \ldots,\left|b_{\ell}\right|\right\}$ and

$$
\Lambda=\alpha_{1}^{b_{1}} \cdots \alpha_{\ell}^{b_{\ell}}-1
$$

Let $A_{1}, \ldots, A_{\ell}$ be real numbers such that

$$
A_{i} \geq \max \left\{d_{\mathbf{L}} h\left(\alpha_{i}\right),\left|\log \alpha_{i}\right|, 0.16\right\} \quad \text { for all } \quad i=1, \ldots, \ell
$$

The following result is due to Matveev in [11] (see also Theorem 9.4 in [4]).

Theorem 2 Assume that $\Lambda \neq 0$. Then

$$
\log |\Lambda|>-1.4 \times 30^{\ell+3} \times \ell^{4.5} \times d_{\mathbf{L}}^{2}\left(1+\log d_{\mathbf{L}}\right)(1+\log B) A_{1} \cdots A_{\ell} .
$$


In this paper, we always use $\ell=3$. Further, $\mathbf{L}=\mathbf{Q}[\sqrt{2}, \sqrt{5}]$ has degree $d_{\mathbf{L}}=4$. Thus, once for all we fix the constant

$$
C:=5.46696 \times 10^{12}>1.4 \times 30^{3+3} \times 3^{4.5} \times 4^{2}(1+\log 4) .
$$

Matveev's bound gives us some large bounds on our parameters. In order to lower such bounds, we use a version of a reduction method of Baker-Davenport based on Lemma in [1]. We shall use the one given by Bravo, Gómez and Luca in [2]. For a real number $x$, we write

$$
\|x\|=\min \{|x-n|: n \in \mathbf{Z}\} .
$$

Lemma 1 Let $M$ be a positive integer. Let $\tau, \mu, A>0, B>1$ be given real numbers. Assume that $p / q$ is a convergent of $\tau$ such that $q>6 M$ and $\varepsilon:=\|q \mu\|-M\|q \tau\|>0$. Then the inequality

$$
0<|n \tau-m+\mu|<\frac{A}{B^{w}}
$$

does not has a solution in positive integers $n, m$ and $w$ in the ranges

$$
n \leq M \quad \text { and } \quad w \geq \frac{\log (A q / \varepsilon)}{\log B} .
$$

This lemma is a slightly variation of the one given by Dujella and Pethő in [9]. The following lemma is also useful. It is Lemma 7 in [10].

Lemma 2 If $m \geq 1, T>\left(4 m^{2}\right)^{m}$ and $T>x /(\log x)^{m}$, then

$$
x<2^{m} T(\log T)^{m} .
$$

\section{Proof of Theorem 1}

We start with some basic properties of our sequences. Put

$$
\alpha:=\frac{1+\sqrt{5}}{2}, \quad \beta:=\frac{1-\sqrt{5}}{2} ; \quad \text { and } \quad \gamma:=1+\sqrt{2}, \quad \delta:=1-\sqrt{2} .
$$

We have the well-known Binet's formulas

$$
F_{n}=\frac{\alpha^{n}-\beta^{n}}{\sqrt{5}} \quad \text { and } \quad P_{n}=\frac{\gamma^{n}-\delta^{n}}{2 \sqrt{2}}
$$

which hold for all $n \geq 0$. Further, the inequalities

$$
\alpha^{n-2} \leq F_{n} \leq \alpha^{n-1} \quad \text { and } \quad \gamma^{n-2} \leq P_{n} \leq \gamma^{n-1}
$$

also hold for all $n \geq 1$.

Now, we start to study our equation (2) in non-negative integers $\left(n, m, n_{1}, m_{1}\right)$ with $(n, m) \neq\left(n_{1}, m_{1}\right)$. As we said, we assume $n \neq 1, n_{1} \neq 1$. It could happen that $\min \left\{n, n_{1}\right\}=0$. At any rate, $\max \left\{n, n_{1}\right\} \geq 2$. If in (2) we have $m=m_{1}$, then $F_{n}=F_{n_{1}}$, 
implies that $n=n_{1}$, a contradiction. Thus, from now on we assume $m>m_{1}$. Rewriting (2) as

$$
F_{n}-F_{n_{1}}=P_{m}-P_{m_{1}},
$$

we observe the right-hand side is positive. Hence, so is the left-hand side, therefore $n>n_{1}$. We now compare the two sides of (5) using (4). We have

$$
\alpha^{n-4} \leq F_{n}-F_{n_{1}}=P_{m}-P_{m_{1}} \leq P_{m} \leq \gamma^{m-1}
$$

The left-hand side inequality is clear if $n_{1}=0$. It is also clear if $n_{1} \neq 0$, since in that case $n_{1} \geq 2$, so $n \geq 3$, so $F_{n}-F_{n_{1}} \geq F_{n}-F_{n-1}=F_{n-2} \geq \alpha^{n-4}$. Thus, $\alpha^{n-4} \leq \gamma^{m-1}$. In a similar way,

$$
\alpha^{n-1} \geq F_{n} \geq F_{n}-F_{n_{1}}=P_{m}-P_{m_{1}} \geq P_{m-1} \geq \gamma^{m-3},
$$

where the right-most inequality is clear (both for $m_{1}=0$ and for $m_{1}>0$ ). We thus have

$$
n-4 \leq(m-1) \frac{\log \gamma}{\log \alpha} \quad \text { and } \quad n-1 \geq(m-3) \frac{\log \gamma}{\log \alpha} .
$$

Since $\log \gamma / \log \alpha=1.831570923 \ldots$ it follows that if $n \leq 300$, then $m \leq 166$. Running a Mathematica program in the range $0 \leq n_{1}<n \leq 300$ and $0 \leq m_{1}<m \leq 166$, with our convention, we obtain all the possibilities listed in Theorem 1.

From now on, $n>300$. Further, by (6) we get $m>162$ and also $n>m$. From Binet's formulas (3), we obtain

$$
\begin{aligned}
\left|\frac{\alpha^{n}}{\sqrt{5}}-\frac{\gamma^{m}}{2 \sqrt{2}}\right| & =\left|\frac{\alpha^{n_{1}}+\beta^{n}-\beta^{n_{1}}}{\sqrt{5}}-\frac{\gamma^{m_{1}}-\delta^{m_{1}}+\delta^{m}}{2 \sqrt{2}}\right| \leq \frac{\alpha^{n_{1}}+2}{\sqrt{5}}+\frac{\gamma^{m_{1}}+2}{2 \sqrt{2}} \\
& \leq 2 \max \left\{\alpha^{n_{1}+2}, \gamma^{m_{1}+1}\right\} .
\end{aligned}
$$

Dividing through by $\gamma^{m} / 2 \sqrt{2}$ we get

$$
\left|\frac{4}{\sqrt{10}} \gamma^{-m} \alpha^{n}-1\right| \leq \max \left\{\alpha^{n_{1}-n+9}, \gamma^{m_{1}-m+3}\right\},
$$

where we have used that $\alpha^{n-4} \leq \gamma^{m-1}$ as well as the fact that $4 \sqrt{2}<\gamma^{2}<\alpha^{4}$. Let $\Lambda$ be the expression inside the absolute value in in the left-hand side above. Observe that $\Lambda$ is not zero. Indeed, otherwise $8 / 5=\gamma^{2 m} / \alpha^{2 n}$ is both a unit (an algebraic integer whose reciprocal is also an algebraic integer) and a rational number, which is false since the only rational units are \pm 1 .

Now we apply Matveev's inequality with

$$
\alpha_{1}=\frac{4}{\sqrt{10}}, \quad \alpha_{2}=\gamma, \quad \alpha_{3}=\alpha, \quad b_{1}=1, \quad b_{2}=-m, \quad b_{3}=n .
$$

We have $B=n$. Further, we have $h\left(\alpha_{1}\right)=\log 8 / 2, h\left(\alpha_{2}\right)=\log \gamma / 2$ and $h\left(\alpha_{3}\right)=$ $\log \alpha / 2$. Thus, we may take $A_{1}:=4.2, A_{2}:=1.8$ and $A_{3}:=1$ we obtain that

$$
\log |\Lambda|>-C(1+\log n) \times 4.2 \times 1.8
$$


Comparing with (8) we obtain

$$
\min \left\{\left(n-n_{1}-9\right) \log \alpha,\left(m-m_{1}-3\right) \log \gamma\right\} \leq 4.13302 \times 10^{13}(1+\log n) .
$$

We next study each of these two possibilities.

Case 1. $\min \left\{\left(n-n_{1}\right) \log \alpha,\left(m-m_{1}\right) \log \gamma\right\}=\left(n-n_{1}\right) \log \alpha$.

To this case, we rewrite our equation as follows:

$$
\begin{aligned}
\left|\left(\frac{\alpha^{n-n_{1}}-1}{\sqrt{5}}\right) \alpha^{n_{1}}-\frac{\gamma^{m}}{2 \sqrt{2}}\right| & =\left|\frac{\beta^{n}-\beta^{n_{1}}}{\sqrt{5}}-\frac{\gamma^{m_{1}}-\delta^{m_{1}}+\delta^{m}}{2 \sqrt{2}}\right| \\
& \leq \frac{2}{\sqrt{5}}+\frac{\gamma^{m_{1}}+2}{2 \sqrt{2}}<\gamma^{m_{1}+2} .
\end{aligned}
$$

Thus,

$$
\left|\left(\frac{4\left(\alpha^{n-n_{1}}-1\right)}{\sqrt{10}}\right) \alpha^{n_{1}} \gamma^{-m}-1\right|<\gamma^{m_{1}-m+4} .
$$

Let $\Lambda_{1}$ be the expression inside the absolute value which is in the left-hand side. We note that $\Lambda_{1} \neq 0$, for if this is not so then we would get

$$
\frac{\alpha^{n}-\alpha^{n_{1}}}{\gamma^{m}}=\frac{\sqrt{10}}{4}
$$

which implies that the right-hand side is an algebraic integer, which it isn't (it's square is 5/8). We apply again Matveev's inequality by taking

$$
\alpha_{1}=\frac{4\left(\alpha^{n-n_{1}}-1\right)}{\sqrt{10}}, \quad \alpha_{2}=\gamma, \quad \alpha_{3}=\alpha, \quad b_{1}=1, \quad b_{2}=-m, \quad b_{3}=n_{1} .
$$

Thus, $B=n$. The heights of $\alpha_{2}$ and $\alpha_{3}$ have already been calculated. As for $h\left(\alpha_{1}\right)$, we have

$$
\begin{aligned}
h\left(\frac{4\left(\alpha^{n-n_{1}}-1\right)}{\sqrt{10}}\right) & \leq h\left(\frac{4}{\sqrt{10}}\right)+h\left(\alpha^{n-n_{1}}-1\right) \leq \frac{\log 8}{2}+h\left(\alpha^{n-n_{1}}\right)+\log 2 \\
& =\frac{\log 32}{2}+\left(n-n_{1}\right) \frac{\log \alpha}{2} \leq \frac{4.13304 \times 10^{13}(1+\log n)}{2},
\end{aligned}
$$

where we have used (9). Thus, we can take $A_{1}:=8.26608 \times 10^{13}(1+\log n), A_{2}$ and $A_{3}$ as in the analysis of $\Lambda$, and get

$$
\log \left|\Lambda_{1}\right|>-C \times\left(8.26608 \times 10^{13}(1+\log n)^{2}\right) \times 1.8 .
$$

Combining this with (10), we get

$$
\left(m-m_{1}\right) \log \gamma<8.13427 \times 10^{26}(1+\log n)^{2} .
$$

Case 2. $\min \left\{\left(n-n_{1}\right) \log \alpha,\left(m-m_{1}\right) \log \gamma\right\}=\left(m-m_{1}\right) \log \gamma$. 
Here, we rewrite our equation as

$$
\begin{aligned}
& \left|\frac{\alpha^{n}}{\sqrt{5}}-\left(\frac{\gamma^{m-m_{1}}-1}{2 \sqrt{2}}\right) \gamma^{m_{1}}\right|=\left|\frac{\beta^{n}+\alpha^{n_{1}}-\beta^{n_{1}}}{\sqrt{5}}-\frac{\delta^{m}-\delta^{m_{1}}}{2 \sqrt{2}}\right| \\
& \leq \frac{\alpha^{n_{1}}+2}{\sqrt{5}}+\frac{1}{\sqrt{2}}<\alpha^{n_{1}+5} .
\end{aligned}
$$

Thus,

$$
\left|1-\left(\frac{\sqrt{10}\left(\gamma^{m-m_{1}}-1\right)}{4}\right) \gamma^{m_{1}} \alpha^{-n}\right|<\alpha^{n_{1}-n+7} .
$$

We let $\Lambda_{2}$ be the expression inside the absolute value in the left-hand side. As before, $\Lambda_{2} \neq 0$, for otherwise we get that $8 / 5$ is an algebraic integer, which is false. We apply again Matveev's inequality by taking

$$
\alpha_{1}=\frac{\sqrt{10}\left(\gamma^{m-m_{1}}-1\right)}{4}, \quad \alpha_{2}=\gamma, \quad \alpha_{3}=\alpha, \quad b_{1}=1, \quad b_{2}=m_{1}, \quad b_{3}=-n .
$$

Thus, $B=n$. The heights of $\alpha_{2}$ and $\alpha_{3}$ have already been calculated. As for $h\left(\alpha_{1}\right)$, we have

$$
\begin{aligned}
h\left(\frac{\sqrt{10}\left(\gamma^{m-m_{1}}-1\right)}{4}\right) & \leq h\left(\frac{\sqrt{10}}{4}\right)+h\left(\gamma^{m-m_{1}}-1\right) \\
& \leq \frac{4.13304 \times 10^{13}(1+\log n)}{2}
\end{aligned}
$$

Thus, we can take the same $A_{1}$ as in Case 1 , and so we get the same lower bound for $\log \left|\Lambda_{2}\right|$. Therefore,

$$
\left(n-n_{1}\right) \log \alpha<8.13427 \times 10^{26}(1+\log n)^{2} .
$$

So, we have proved that

$$
\max \left\{\left(n-n_{1}\right) \log \alpha,\left(m-m_{1}\right) \log \gamma\right\} \leq 8.13427 \times 10^{26}(1+\log n)^{2} .
$$

We now get a bound on $n$. Using Binet's formulas (3), we write our equation as follows:

$$
\left|\frac{\alpha^{n-n_{1}}-1}{\sqrt{5}} \alpha^{n_{1}}-\frac{\gamma^{m-m_{1}}-1}{2 \sqrt{2}} \gamma^{m_{1}}\right|=\left|\frac{\beta^{n}-\beta^{n_{1}}}{\sqrt{5}}-\frac{\delta^{m}-\delta^{m_{1}}}{2 \sqrt{2}}\right|<\frac{2}{\sqrt{5}}+\frac{1}{\sqrt{2}}<2 .
$$

Dividing across by $\left(\gamma^{m}-\gamma^{m-1}\right) / 2 \sqrt{2}$, we obtain

$$
\left|\left(\frac{4}{\sqrt{10}}\left(\frac{\alpha^{n-n_{1}}-1}{\gamma^{m-m_{1}}-1}\right)\right) \gamma^{-m_{1}} \alpha^{n_{1}}-1\right|<\frac{4 \sqrt{2}}{\gamma^{m}-\gamma^{m_{1}}}<\frac{8 \sqrt{2}}{\gamma^{m}}<\frac{1}{\alpha^{n-8}},
$$

where we used $\alpha^{n-4}<\gamma^{m-1}$, as well as the fact that $8 \sqrt{2}<\alpha^{4} \gamma$. We let $\Lambda_{3}$ be the expression inside the absolute value in (13). We apply Matveev's inequality with

$$
\alpha_{1}=\frac{4}{\sqrt{10}}\left(\frac{\alpha^{n-n_{1}}-1}{\gamma^{m-m_{1}}-1}\right), \alpha_{2}=\gamma, \alpha_{3}=\alpha, b_{1}=1, b_{2}=-m_{1}, b_{3}=-n_{1} .
$$


Thus, we take $B=n$. We need to show that $\Lambda_{3} \neq 0$. To do this we take the $\mathbf{Q}$ automorphism $\sigma$ of $\mathbf{L}$ given by $\sigma(\sqrt{5})=-\sqrt{5}$ and $\sigma(\sqrt{2})=\sqrt{2}$. Under this automorphism, we have $\sigma(\alpha)=\beta, \sigma(\gamma)=\gamma$ and $\sigma(\sqrt{10})=-\sqrt{10}$. Thus, if $\Lambda_{3}=0$, then $\sigma\left(\Lambda_{3}\right)=0$, which implies, in particular, that

$$
\frac{\sqrt{10}}{4}=\left|\frac{\beta^{n}-\beta^{n_{1}}}{\gamma^{m}-\gamma^{m_{1}}}\right|<\frac{2}{\gamma^{m-1}(\gamma-1)}<\frac{1}{2},
$$

since $m>162$, which is a contradiction. As before, the heights of $\alpha_{2}$ and $\alpha_{3}$ have already been calculated. For $h\left(\alpha_{1}\right)$, we have

$$
\begin{aligned}
h\left(\frac{4}{\sqrt{10}}\left(\frac{\alpha^{n-n_{1}}-1}{\gamma^{m-m_{1}}-1}\right)\right) & \leq h\left(\frac{4}{\sqrt{10}}\right)+h\left(\alpha^{n-n_{1}}+1\right)+h\left(\gamma^{m-m_{1}}+1\right) \\
& \leq \frac{\log 128}{2}+\left(n-n_{1}\right) \frac{\log \alpha}{2}+\left(m-m_{1}\right) \frac{\log \gamma}{2} \\
& \leq 8.13428 \times 10^{26}(1+\log n)^{2} .
\end{aligned}
$$

Thus, we can take $A_{1}:=3.25371 \times 10^{27}(1+\log n)^{2}$, and $A_{2}, A_{3}$ as before. Therefore, we get

$$
\begin{aligned}
\log \left|\Lambda_{3}\right| & >-C(1+\log n) \times\left(3.25371 \times 10^{27}(1+\log n)^{2}\right) \times 1.8 \\
& >-3.20183 \times 10^{40}(1+\log n)^{3}
\end{aligned}
$$

which, upon comparing it to (13) and applying Lemma 2, we obtain

$$
n<3.77671 \times 10^{48} \text {. }
$$

Now, we will reduce the upper bound of $n$. To do this, let $\Gamma$ be defined as

$$
\Gamma=n \log \alpha-m \log \gamma+\log \left(\frac{4}{\sqrt{10}}\right) .
$$

Assume first that $\min \left\{n-n_{1}, m-m_{1}\right\} \geq 20$. We go to (8). Note that $e^{\Gamma}-1=\Lambda \neq 0$, so $\Gamma \neq 0$. If $\Gamma>0$ then

$$
0<\Gamma<e^{\Gamma}-1=\Lambda=|\Lambda|<\max \left\{\alpha^{n_{1}-n+9}, \gamma^{m_{1}-m+3}\right\} .
$$

On the other hand, if $\Gamma<0$, we then have $1-e^{\Gamma}=\left|e^{\Gamma}-1\right|<1 / 2$ which implies $e^{|\Gamma|}<2$. Thus,

$$
0<|\Gamma|<e^{|\Gamma|}-1=e^{|\Gamma|}|\Lambda|<2 \max \left\{\alpha^{n_{1}-n+9}, \gamma^{m_{1}-m+3}\right\} .
$$

So, in both cases we have

$$
0<|\Gamma|<2 \max \left\{\alpha^{n_{1}-n+9}, \gamma^{m_{1}-m+3}\right\} .
$$

Dividing through by $\log \gamma$ in the above inequality, we get

$$
0<|n \tau-m+\mu|<\max \left\{\frac{173}{\alpha^{n-n_{1}}}, \frac{32}{\gamma^{m-m_{1}}}\right\},
$$


where

$$
\tau:=\frac{\log \alpha}{\log \gamma}, \quad \mu:=\frac{\log (4 / \sqrt{10})}{\log \gamma} .
$$

Now we apply Lemma 1 . To do this, we take $M:=3.77671 \times 10^{48}$ (a bound on $m$ and $n$ by (14)), our $\tau$ and, with a Mathematica program, we find that the denominator of the convergent

$$
\frac{p_{112}}{q_{112}}=\frac{111842821415068814601069451383096958405345992106163812}{204848059751598401563305907296432335323118859258712413}
$$

of $\tau$ satisfies $q_{112}>6 M$ and that $\varepsilon=\|q \mu\|-M\|q \tau\|=0.105822>0$. This implies, with $(A, B)=(173, \alpha)$ or $(32, \gamma)$, that either

$$
n-n_{1} \leq 270, \quad \text { or } \quad m-m_{1} \leq 145 .
$$

We now look at each one of these two cases. First, we assume that $n-n_{1} \leq 270$ and $m-m_{1} \geq 20$. In this case, we consider

$$
\Gamma_{1}=n_{1} \log \alpha-m \log \gamma+\log \left(\frac{4\left(\alpha^{n-n_{1}}-1\right)}{\sqrt{10}}\right) .
$$

As before, $e^{\Gamma_{1}}-1=\Lambda_{1} \neq 0$, so $\Gamma_{1} \neq 0$. We go to (10). With an argument similar to a previous one, we have that

$$
0<\left|\Gamma_{1}\right|<\frac{2 \gamma^{4}}{\gamma^{m-m_{1}}}
$$

Dividing through by $\log \gamma$ we obtain

$$
0<\left|n_{1} \tau-m+\mu\right|<\frac{78}{\gamma^{m-m_{1}}},
$$

where $\tau$ is the same one as above and

$$
\mu:=\frac{\log \left(4\left(\alpha^{n-n_{1}}-1\right) / \sqrt{10}\right)}{\log \gamma} .
$$

We apply again Lemma 1 noting that $n_{1}>0$, for otherwise we would have that $n \leq$ 270 which contradicts our hypothesis that $n>300$. Consider

$$
\mu_{k}:=\frac{\log \left(4\left(\alpha^{k}-1\right) / \sqrt{10}\right)}{\log \gamma}, \quad \text { for } \quad k=1, \ldots, 270 .
$$

We ran a Mathematica program and found that the same convergent $p_{112} / q_{112}$ satisfies $q_{112}>6 M$. Further, $\varepsilon_{k} \geq 0.00119532$ for all $1 \leq k \leq 270$. For each of the values of $\varepsilon_{k}$ and with $(A, B)=(78, \gamma)$, we calculate $\log \left(78 q_{112} / \varepsilon_{k}\right) / \log \gamma$ and found that each of them is at most 151 . Thus, $m-m_{1} \leq 151$.

Now let us look at the other case. Assume that $m-m_{1} \leq 145$ and $n-n_{1} \geq 20$. We consider

$$
\Gamma_{2}=n \log \alpha-m_{1} \log \gamma+\log \left(\frac{4}{\sqrt{10}\left(\gamma^{m-m_{1}}-1\right)}\right) .
$$


We note that $1-e^{-\Gamma_{2}}=\Lambda_{2} \neq 0$, so $\Gamma_{2} \neq 0$. We go to (11). With an argument similar to one above, we obtain

$$
0<\left|\Gamma_{2}\right|<\frac{2 \alpha^{7}}{\alpha^{n-n_{1}}} .
$$

Dividing through by $\log \lambda$, we get

$$
0<\left|n \tau-m_{1}+\mu\right|<\frac{66}{\alpha^{n-n_{1}}},
$$

where $\tau$ is the same one as above and

$$
\mu:=\frac{\log \left(4 /\left(\sqrt{10}\left(\gamma^{m-m_{1}}-1\right)\right)\right)}{\log \gamma} .
$$

Now we use again Lemma 1 noting that $m_{1}>0$, which is the case, since otherwise we have $m \leq 145$, which contradicts our hypothesis $m>162$. As above, by considering now

$$
\mu_{\ell}:=\frac{\log \left(4 /\left(\sqrt{10}\left(\gamma^{\ell}-1\right)\right)\right)}{\log \gamma}, \quad \text { for all } \quad \ell=1, \ldots, 145
$$

and running a Mathematica program, we find that $q_{112}>6 M$, and that for this convergent $\varepsilon_{\ell} \geq 0.0000620746$ for all $1 \leq \ell \leq 145$. For each of these $\varepsilon_{\ell}$ and with $(A, B):=$ $(66, \alpha)$, we calculated $\log \left(66 q_{112} / \varepsilon_{\ell}\right) / \log \alpha$ and found that all these numbers are at most 283. Thus $n-n_{1} \leq 283$.

So, we got that either $n-n_{1} \leq 270$ or $m-m_{1} \leq 145$. Assuming the first one we deduced $m-m_{1} \leq 151$, and assuming the second one, we deduced $n-n_{1} \leq 283$. Altogether, we have $n-n_{1} \leq 283, m-m_{1} \leq 151$. So, it remains to study this case. We consider

$$
\Gamma_{3}=n_{1} \log \alpha-m_{1} \log \gamma+\log \left(\frac{4}{\sqrt{10}}\left(\frac{\alpha^{n-n_{1}}-1}{\gamma^{m-m_{1}}-1}\right)\right) .
$$

We note that $e^{\Gamma_{3}}-1=\Lambda_{3}$. Again, since $\Lambda_{3} \neq 0$, we have that $\Gamma_{3} \neq 0$. Since $n>300$, we get

$$
0<\left|\Gamma_{3}\right|<\frac{2 \alpha^{8}}{\alpha^{n}}
$$

Dividing through by $\log \gamma$, we get

$$
0<\left|n_{1} \tau-m_{1}+\mu\right|<\frac{107}{\alpha^{n}}
$$

where $\tau$ is as above and

$$
\mu:=\frac{\log \left(4\left(\alpha^{n-n_{1}}-1\right) / \sqrt{10}\left(\gamma^{m-m_{1}}-1\right)\right)}{\log \gamma} .
$$

We apply for the last time Lemma 1 . As above, we have that $n_{1}, m_{1}>0$. Thus, we consider

$$
\mu_{k, \ell}:=\frac{\log \left(4\left(\alpha^{k}-1\right) / \sqrt{10}\left(\gamma^{\ell}-1\right)\right)}{\log \gamma}, \quad k=1, \ldots, 283, \quad \ell=1, \ldots, 151 .
$$


Running a Mathematica program, we find again that the same convergent works namely $q_{112}>6 M$ and $\varepsilon_{k, \ell} \geq 0.0000307767$ for all $1 \leq k \leq 283$ and $1 \leq \ell \leq 151$. With $(A, B):=(107, \alpha)$ we calculated $\log \left(107 q_{112} / \varepsilon_{k, \ell}\right) / \log \alpha$ for each of these values $\varepsilon_{k, \ell}$, and found that the maximum value of them is $\leq 286$. Thus, $n \leq 286$, which contradicts our assumption on $n$.

This finishes the proof of our theorem.

Acknowledgements We thank the referees for their comments and remarks which improve the quality of this work. S.H.H. thanks Leticia A. Ramírez for generous support. F. L. was supported in part by grant CPRR160325161141 and an A-rated scientist award both from the NRF of South Africa and by grant no. 17-02804S of the Czech Granting Agency. F.L. worked on this paper during visits at the Mathematics Department to the UAZ, Zacatecas, Mexico and the Max Planck Institute for Mathematics, Bonn, Germany in February and March of 2018, respectively. He thanks these institutions for the generous support and hospitality. L.M.R. was partially supported by grant PFCE 2018-2019 (UAZ-CA-169).

\section{References}

1. A. BAKer, H. DAVEnport, The equations $3 X^{2}-2=Y^{2}$ and $8 X^{2}-7=Z^{2}$, Quart. J. Math. Oxford (20) (2), 129-137, 1969

2. J.J. Bravo, C. A. Gómez, F. LuCA, Powers of two as sums of two $k$-Fibonacci numbers, Miskolc Math. Notes (17) (1), 85-100, 2016.

3. J.J. BRAVO, F. LuCA, K. YAZÁn, On Pillai's problem with Tribonacci numbers and Powers of 2, Bull. Korean Math. Soc, (54) (3), 1069-11080, 2017.

4. Y. Bugeaud, M. Mignotte, S. Siksek, Classical and modular approaches to exponential diophantine equations I: Fibonacci and Lucas perfect powers, Ann. of Math. 163, 969-1018, 2006.

5. K.C. Chim, I. Pink, V. ZIEgLer, On a varian of Pillai's problem, Int. J. Number Theory (7), 17111727, 2017.

6. K.C. Chim, I. Pink, V. Ziegler, On a varian of Pillai's problem II, J. Number Theory (183), 269290, 2018

7. M. Ddamulira, C. A. Gómez, F. LuCA, On a problem of Pillai with $k$-generalized Fibonacci numbers and powers of 2, Monatsh. Math. (2018). https://doi.org/10.1007/s00605-018-1155-1

8. M. Ddamulira, F. LuCA, M. Rakotomalala, On a problem of Pillai with Fibonacci and powers of 2, Proc. Indian Acad. Sci. (Math. Sci.) (127) (3), 411-421, 2017.

9. A. Dujella, A. Pethô, A generalization of a theorem of Baker and Davenport, Quart. J. Math. Oxford (49) (3), 291-306, 1998.

10. S. GUZMÁn SÁNCHEZ, F. LUCA, Linear combinations of factorials and $S$-units in a binary recurrence sequence, Ann. Math. Québec (38), 169-188, 2014.

11. E. M. MAtveEv, An explicit lower bound for a homogeneous rational linear form in the logarithms of algebraic numbers II, Izv. Math textbf(64) (6), 1217-1269, 2000.

12. S. S. Pillai, On $a^{x}-b^{y}=c$, J. Indian Math. Soc., (2), 119-122 (1936). 